\title{
Multiplexing holograms for data page storage using a LCD as a hybrid ternary modulation
}

\author{
Elena Fernández ${ }^{(1) *}$, Manuel Ortuño ${ }^{(2)}$, Sergi Gallego ${ }^{(2)}$, Celia García $^{(1)}$, Andrés Márquez $^{(2)}$, \\ Augusto Beléndez ${ }^{(2)}$ and Inmaculada Pascual ${ }^{(1)}$ \\ ${ }^{11}$ Dep. de Óptica, Farmacología y Anatomía \\ ${ }^{(2)}$ Dept. de Física, Ing. de Sistemas y Ta de la Señal \\ Universidad de Alicante, Apartado 99, E-03080 Alicante, Spain \\ *Email: Elena.Fernandez@ua.es
}

\begin{abstract}
Holographic data storage is a new optical technology which allows an important number of bits to be stored in a recording material. In this work, holographic data pages were stored in a PVA/acrylamide photopolymer layer using a peristrophic multiplexing method. This material is formed of acrylamide photopolymers, which are considered interesting materials for recording holographic memories. A liquid crystal (LCD) device was used to modify the object beam and store the data pages in the material. This LCD was used to modulate the object beam in order to reduce the zero frequency of the Fourier Transform (FT) of the object. The scheme used to reduce this frequency was hybrid ternary modulation (HTM).
\end{abstract}

Keywords: Photopolymers, Holographic recording materials, Liquid crystal device, Hybrid ternary modulation.

\section{INTRODUCTION}

Two-dimensional memory technologies like CD-ROM and DVD have reached the limits of their capacity and so there is a need for new technological systems capable of storing more information. Holographic data storage (threedimensional technology) is a new optical memory technology that allows an important number of bits to be stored in a recording material with greater capacity, higher density and faster readout rates than two-dimensional technology.

A photopolymerizable material was used to store the holograms. These materials have excellent holographic characteristics, such as high refractive index modulation ${ }^{1,2}$, large dynamic range ${ }^{3-5}$, good light sensitivity, real time image development, high optical quality, low cost, and have been used as the basis of new 3-D holographic disks. In addition to this, their properties like energetic sensitivity or spectral sensitivity can be easily changed by modifying their composition ${ }^{\mathbf{1 , 3 6}}$. The photopolymer is composed of acrylamide (AA) as the polymerizable monomer, triethanolamine (TEA) as radical generator, N,N'methylene-bis-acrylamide (BMA) as crosslinker, yellowish eosin (YE) as sensitizer and a binder of polyvinyl alcohol (PVA). Layers of this material about $80 \mu \mathrm{m}$ thick were used to store the holograms.

Twisted-nematic liquid crystal displays (TN-LCDs) have been used in recent years as spatial light modulators (SLMs) to modify in real time the amplitude or phase of a light beam ${ }^{7-10}$. This LCD can be used to design programmable optical elements, such as lenses and data pages or in holographic data storage.

* Elena.Fernandez@ua.es; phone +34 965903400 Ext 2886; fax +34 965903746

Holography: Advances and Modern Trends, edited by Miroslav Miler, Miroslav Hrabovský,

Proc. of SPIE Vol. 7358, 73581C · C 2009 SPIE · CCC code: 0277-786X/09/\$18 - doi: 10.1117/12.820622 
In holographic data storage systems (HDSS), LCDs are normally used as binary amplitude transparencies to display the data page, and the Fourier transform (FT) of the data page is then holographically recorded on a photosensitive material. However, amplitude modulation produces a high zero frequency which may saturate the dynamic range of the material thus limiting the accessible dynamic range. The problems caused by the lack of homogeneity of the FT can be solved by using some other modulation schemes, such as random phase masks ${ }^{11}$, binary $\pi$ radians phase-only modulation $(\pi \mathrm{BPM})$ ${ }^{12-15}$ or hybrid ternary modulation (HTM) ${ }^{13,15-18}$.

In this study the data pages are sent to the LCD and stored in the material using HTM. In this method, some bits are represented in the data page by two different gray levels that produce a maximum transmission and have a phase difference of $\pi$ rad (the "one" bits). The other bits are represented by a gray level with a minimum transmission (the "zero" bits). Using this method, if half of the bits with the maximum intensity have a phase difference of $\pi \mathrm{rad}$ compared with the other half, the intensity of the high zero frequency of the FT can be reduced ${ }^{15}$.

Moreover many holograms were superimposed at the same position in the material using peristrophic multiplexing in order to store information with holographic techniques, and the object beam was modulated using HTM so as to reduce the zero frequency.

Different objects were stored in the photopolymer. Once the hologram was stored, it was illuminated in the reconstruction stage by the same plane wave as in the recording process in order to prevent the appearance of aberrations in the reconstructed image. Using an optical system, the stored information was imaged onto a CCD camera connected to a personal computer, where the images were analyzed and processed.

Once the images have been obtained, a criterion is needed to assess the quality of the different images and to compare them with the original object. In order to evaluate the image quality, its histogram was used to calculate the bit error rate (BER) ${ }^{19-21}$ and determine the contrast between the pixels. BER values of each image were calculated to decide which parameters provide the best image quality (greater contrast and less noise).

\section{EXPERIMENTAL}

\subsection{Preparation of the material}

The photopolymer in which the holograms were recorded was composed of acrylamide (AA) as the polymerizable monomer, triethanolamine (TEA) as radical generator, N,N'methylene-bis-acrylamide (BMA) as crosslinker, yellowish eosin (YE) as sensitizer and a binder of polyvinyl alcohol (PVA). Table 1 shows the component concentrations of the photopolymer composition used to obtain layers about $80 \mu \mathrm{m}$ thick.

Table 1: Concentrations of the photopolymer composition.

\begin{tabular}{|cc|}
\hline & Composition \\
\hline Polyvinylalcohol & $6.6 \% \mathrm{w} / \mathrm{v}$ \\
Acrylamide & $0.33 \mathrm{M}$ \\
Triethanolamine & $0.17 \mathrm{M}$ \\
Yellowish eosin & $2.4 \cdot 10^{-4} \mathrm{M}$ \\
N,N'methylene-bis-acrylamide & $0.027 \mathrm{M}$ \\
\hline
\end{tabular}

A solution of PVA in water forms the matrix and this is used to prepare the mixture of AA, BMA, and photopolymerization initiator system composed of TEA and YE. The mixture is made under red light, deposited by gravity on a $22 \times 40 \mathrm{~cm}$ glass plate and left in the dark for one day to allow the water to evaporate in conditions of temperature, $\mathrm{T}$, between $20{ }^{\circ} \mathrm{C}$ and $25{ }^{\circ} \mathrm{C}$, and relative humidity, $\mathrm{RH}, 40 \%-60 \%$. These conditions of drying time, temperature and relative humidity are optimized to obtain the maximum diffraction efficiency of the gratings. Once dry, the glass is cut into squares of $5 \times 5 \mathrm{~cm}$.

\subsection{Holographic set-up}

Holographic data pages were recorded using a diode-pumped frequency-doubled $\mathrm{Nd} \mathrm{YVO}_{4}$ laser (Coherent Verdi V2). The output of the laser was split into two beams, the object and de reference beam. The two beams were spatially 
filtered, using a microscope objective lens and a pinhole, and collimated to yield a plane-wave source of light at 532 $\mathrm{nm}$. The diameter of these beams was $1.5 \mathrm{~cm}$. The two laser beams were spatially overlapped at the recording medium intersection at an angle of $17.4^{\circ}$ (measured in air).

An LCD was placed in the object beam between two polarizers, one to each side of the LCD. The LCD and polarizers were used as an SLM. In addition, a lens was placed in front of the SLM to do the Fourier transform (FT) of the data page which was sent to the SLM. A diaphragm was placed just in front of the photopolymer to block all the orders that leave the LCD except the central order. If the other orders were not blocked, they would also be stored in the material, and during reconstruction interference patterns would be observed on the image, thus worsening its quality. The reference beam was a plane wave that interferes with the object beam at the surface of the material. In previous papers we studied how the beam ratio between object beam and reference beam affects the quality of the stored images. These beam intensities were measured at the position where the photopolymer must be placed when the holograms are stored. In the reconstruction stage, the stored hologram that contains the information of the data page was illuminated with the reference beam, but at a very low intensity so as not to deform the hologram, because the material is sensitive to this wavelength. Another lens was placed behind the photopolymer to do the inverse Fourier transform (IFT) of the diffracted beam on the surface of the charge coupled device (CCD). A computer sent the data pages to the LCD and captured images reconstructed by the CCD.

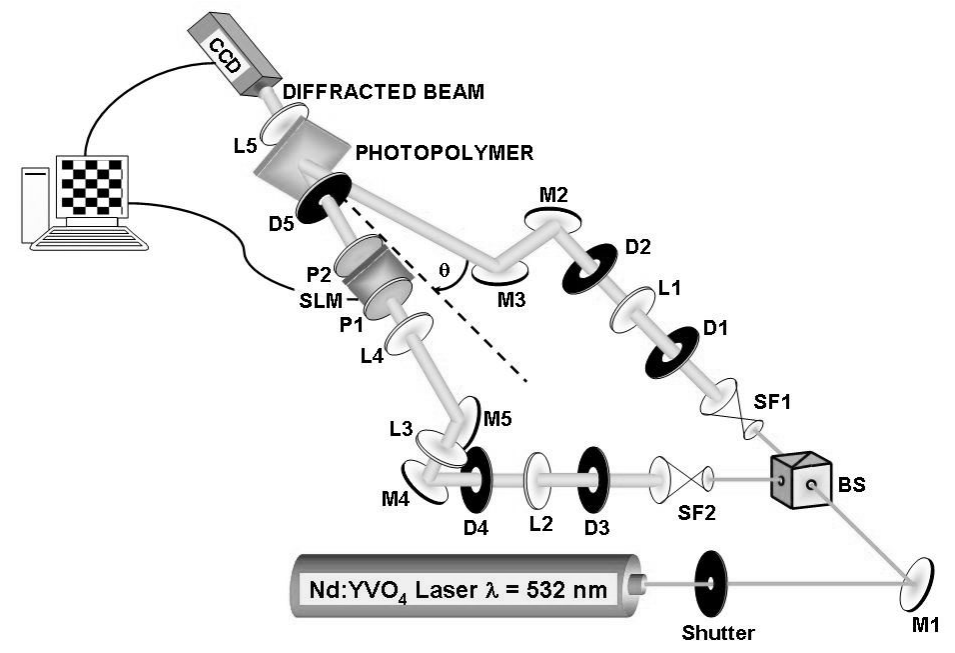

Figure 1: Experimental setup: BS beam splitter, Mi mirror, Li lens, Di, diaphragm, SFi, microscope objective lens and pinhole, SLM spatial light modulator, Pi polarizer, CCD charge coupled device.

\section{RESULTS}

In this paper holograms were multiplexed in layers about $80 \mu \mathrm{m}$ thick using the composition in Table 1 . Binary data pages with a random pixel form were stored. These objects have a different number of pixels: 300x300, 400x400, $500 \times 500$ and $800 \times 600$. This allows us to study the behaviour of the photopolymer material when data pages with different numbers of pixels are multiplexed.

These objects were stored at the same position in the material using peristrophic multiplexing because previous papers showed that when a not very large number of holograms (fewer than 200) are stored, higher diffraction efficiency is obtained with peristrophic multiplexing ${ }^{22}$.

The holograms were stored with an angular separation of $3^{\circ}$. The angular selectivity of the layers was measured in this study and, for a thickness of $80 \mu \mathrm{m}$, we obtained an angular selectivity of $2^{\circ}$. Therefore, an angular separation of $3^{\circ}$ is more than sufficient to prevent the holograms from overlapping. Moreover, the holograms were recorded with a 
reference to object beam ratio of 100 and a reading beam intensity of $0.03 \mathrm{~mW} / \mathrm{cm}^{2}$. In previous studies of the influence of these parameters on the quality of the stored images ${ }^{23,24}$, these values were found to give a greater image quality and, therefore, a lower BER.

\subsection{Optimization of the LCD}

As described in the Holographic set-up section, an LCD was placed in the object beam to modify the wavefront and store this variation in the photopolymer. The variation may be in phase, amplitude or both. In the Introduction section we explained that HTM was used to reduce the zero frequency of the FT. In order to use HTM we need to obtain three states with the LCD: two states with maximum transmission and a phase difference of $\pi \mathrm{rad}$, and a third state with minimum transmission. However, to achieve this, the LCD and polarizers must be calibrated correctly ${ }^{8,10,25}$, which means that the angles at which the latter are to be placed must be optimized in order to obtain the states.

The calibration process consists of two calibration steps. In the first step, the LCD is turned off and no voltage is applied. In this step, three parameters that are independent of the voltage are calculated: the total twist angle $(\alpha)$, the orientation of the molecular director at the input face $\left(\Psi_{\mathrm{D}}\right)$ and the maximum birefringence $\left(\beta_{\max }\right)$. The parameters obtained with the LCD calibrated while turned off are shown in table 2.

Table 2: LCD Calibration parameters independent of the voltage applied.

\begin{tabular}{|ccccc|}
\hline$\alpha$ & $\Psi_{D}$ & $\beta_{\max }\left(\lambda_{0}=633 \mathrm{~nm}\right)$ & $\beta_{\max }\left(\lambda_{0}=532 \mathrm{~nm}\right)$ & $\beta_{\max }\left(\lambda_{0}=442 \mathrm{~nm}\right)$ \\
\hline $94^{\mathrm{o}} \pm 1^{\mathrm{o}}$ & $45^{\mathrm{o}} \pm 1^{\mathrm{o}}$ & $118^{\circ} \pm 1^{\circ}$ & $168^{\circ} \pm 1^{\circ}$ & $199^{\circ} \pm 1^{\mathrm{o}}$ \\
\hline
\end{tabular}

In the second step, the parameters dependent on voltage are measured. These are related to the variation in optical anisotropic properties throughout the thickness of the cell as a function of the voltage applied. The model attempts to take into account the fact that the liquid crystal molecules near the glass are practically adhered to its surface and cannot reorientate themselves when the voltage is applied. Thus, the total thickness $d$ of the LCD may be decomposed into two lateral regions of width $d_{1}$ and a central region of width $d_{2}$. In this way, the anisotropic properties of the LCD may be modeled using two voltage-dependent parameters - birefringence $\beta$ and $\delta$ - which are expressed in Eq. 1 .

$$
\begin{aligned}
& \beta(V)=\pi \Delta n d_{2} / \lambda_{0} \\
& \delta(V)=\pi \Delta n_{\max } d_{1} / \lambda_{0}
\end{aligned}
$$

where $\lambda_{0}$ is the wavelength of the light, $\Delta \mathrm{n}$ is the difference between the ordinary and extraordinary index, with $\Delta \mathrm{n}_{\max }$ being the maximum value.

From the curves of $\beta(\mathrm{V})$ and $\delta(\mathrm{V})$, we can find the angles at which the polarizers must be placed in the experimental setup to modulate the incident beam. In our study, our aim is to obtain the maximum contrast between maximum intensity bits and minimum intensity bits, there must be a phase difference of $\pi$ rad between half of the bits with maximum intensity and the other half. To find these states, the two figures of merit in Eq. 2 were combined.

$$
\begin{gathered}
f_{1}=\frac{I_{\max }-I_{\min }}{I_{\max }+I_{\min }} \\
f_{2}=\frac{M a x[\text { phase }]-M i n[\text { phase }]}{360}
\end{gathered}
$$

The configuration found to satisfy the two figures of merit is shown in Figs. 1 and 2. This configuration was obtained with the angles $\varphi_{1}=178^{\circ}$ for the polarizer in front of the LCD and $\varphi_{2}=93^{\circ}$ for the polarizer behind it.

In Fig. 2a black circles represent the intensity that can be obtained in the configuration with the angles $\varphi_{1}$ and $\varphi_{2}$ depending on the gray level. White circles represent intensity imposed by the figure of merit. In Fig. $2 \mathrm{~b}$ black circles represent the phase obtained depending on the gray level, and white circles represent the phase imposed by the figure of merit. With this configuration, the three states chosen to apply HTM have been rounded in Fig. 2. These three states are summarized in Table 3. 


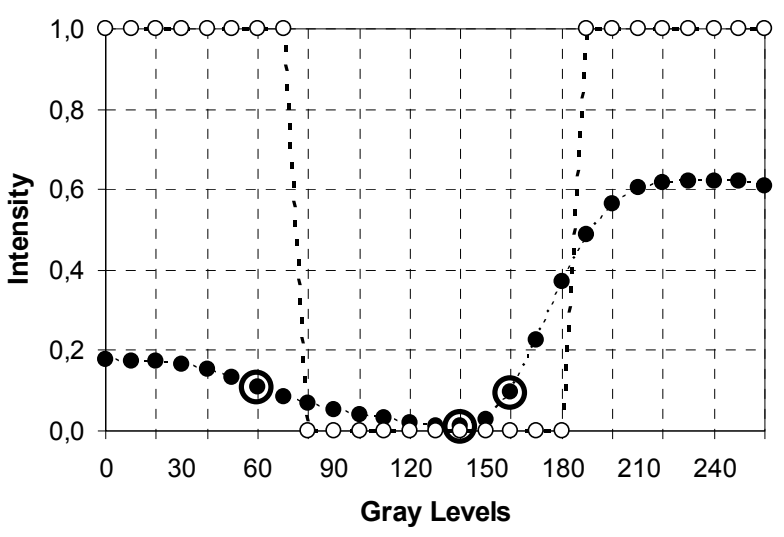

a)

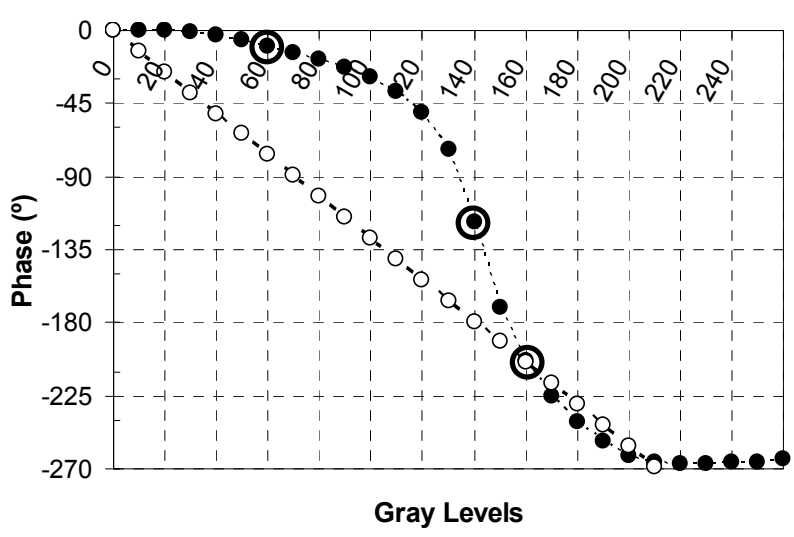

b)

Figure 2: a) Black circles represent the intensity obtained with the polarizers at angles $\varphi_{1}=178^{\circ}$ and $\varphi_{2}=93^{\circ}$, and white circles, the intensity imposed by the figure of merit. b) Black circles represent the intensity obtained with the polarizers at angles $\varphi_{1}=178^{\circ}$ and $\varphi_{2}=93^{\circ}$, and white circles, the intensity imposed by the figure of merit.

Table 3: Gray level, intensity and phase of the states obtained with $\varphi_{1}=178^{\circ}$ and $\varphi_{2}=93^{\circ}$.

\begin{tabular}{|cccc|}
\hline & White 1 & White 2 & Black \\
\hline Gray Level & 60 & 160 & 140 \\
Intensity & 0.11 & 0.10 & 0.01 \\
Phase & $9^{\circ}$ & $204^{\circ}$ & $118^{\circ}$ \\
\hline
\end{tabular}

Fig. 3a represents a model of the data page which was created with the gray levels in Table 3 . Fig. $3 b$ was obtained when the data page in Fig. 3a was sent to the LCD. The angles of the polarizers were $\varphi_{1}=178^{\circ}$ and $\varphi_{2}=93^{\circ}$ in order to obtain the HTM configuration. They were illuminated with the laser and a CCD camera captured the image formed.
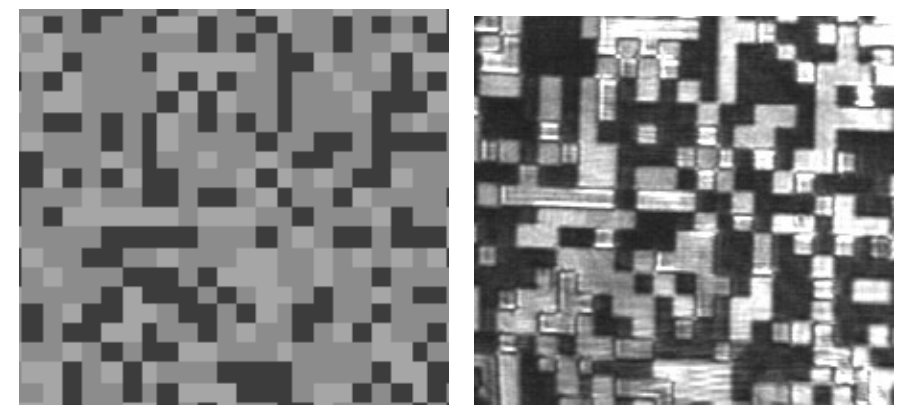

Figure 3: a) Data page created with the gray levels 60, 160 and 140. b) Image obtained with the HTM configuration.

\subsection{Thickness of $80 \mu \mathrm{m}$.}

In section 3.1 the SLM was optimized for storing data pages with the HTM method, which reduces the zero order of the $\mathrm{TF}$ of the objects. The data pages mentioned in that section were stored in an $80 \mu \mathrm{m}$ thick material with four different pixel sizes: 300x300, 400x400, 500x500 and 800x600. Each of the four objects was multiplexed at the same position in the material with an angular separation of $3^{\circ}$ by peristrophic multiplexing. In addition, they were stored with a beam ratio of 100 and reconstructed on the CCD with a reading beam intensity of $0.03 \mathrm{~mW} / \mathrm{cm}^{2} 24$. As the holograms are 
stored in the material, the dynamic range is consumed ${ }^{19-22}$; therefore, the exposure must be increased in order to store more holograms. Of course, when all the dynamic range is consumed, no more holograms will be formed in the material even if the exposure is increased.

After one of the objects has been stored in the photopolymer, the hologram formed is reconstructed illuminating it with the reference beam. The diffractive beam obtained is imaged onto the CCD. However, the image may be distorted for many reasons; therefore, it is advisable to measure a parameter that quantifies the image quality. This parameter is the bit error rate (BER) which is defined as the probability of having erroneous bits in the image. References $21,23,24,26$ explain how to calculate the BER.

Data pages are multiplexed with the four bit sizes with the exposure represented in Figure 4 by purple triangles as a function of the number of holograms. First data pages with 300x300 bits were stored with the exposure shown in Figure 4. Once stored the holograms were illuminated with a reference beam intensity of $0.3 \mathrm{~mW} / \mathrm{cm}^{2}$. The reconstructed images were captured by a CCD camera.

The next step was to calculate the BER of the images. The BER results obtained for the $300 \times 300$ bit object are represented in Figure 4 by solid black circles as a function of the number of holograms. As can be seen, 22 holograms with a BER less than 0.2 were stored. When more holograms are stored the image is almost all noise. The first seven holograms have a BER very close to zero. From hologram 8 on, the BER increases slowly so that holograms 8 to 21 have a BER of around 0.1 . Holograms 22 and 23 have a BER of approximately 0.2 and the other holograms have a BER of above 0.2 .

The next object to be multiplexed was the $400 \times 400$ bit object. It was stored with the same exposure represented by solid purple triangles in Fig. 4. Once stored, the holograms were reconstructed with the reference beam, the images were captured by the CCD camera and their BER was calculated. BER values obtained for this object are shown in Figure 4 (empty blue circles). In this case 25 holograms with a BER less than or equal to 0.2 were obtained.

Then, the 500x500 bit object was stored in the photopolymer. The BER of the images corresponding to this object is represented in Fig. 4 by solid red squares. In this case 25 holograms were also obtained with a BER of less than 0.15 . These holograms have practically the same BER as the 400x400 bit object.

And finally, the 800x600 bit object was multiplexed in the material and the BER values obtained are represented in Fig. 4 by empty green squares. As in the previous cases, the first seven holograms have a BER very close to zero. However, the other holograms have a slightly higher BER. The BER value for holograms 8 to 23 is around 0.2 . In this case, 23 holograms were stored.

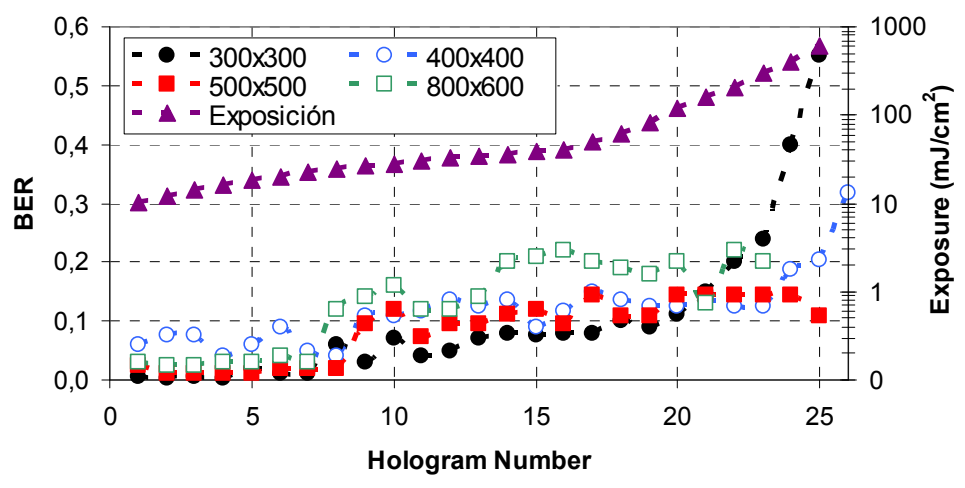

Fig. 4: BER of the $300 \times 300$ pixel (solid black circles), 400x400 pixel (empty blue circles), 500x500 pixel (solid red squares) and 800x600 pixel (empty green squares) objects in a $80 \mu \mathrm{m}$ thick material stored with the HTM configuration, and exposure (solid purple triangles). 
Figure 5 shows the images with the lowest BER for each object. Fig. 5a shows the image of hologram 1 of the $300 \times 300$ pixel object which has a BER $=0.01$; Fig. $5 \mathrm{~b}$ represents the image of hologram 4 of the $400 \times 400$ pixel object with a $\mathrm{BER}=0.03$; Fig. $5 \mathrm{c}$ shows hologram 1 of the $500 \times 500$ pixel object with a $\mathrm{BER}=0.02$, and Fig. $5 \mathrm{~d}$ shows hologram 1 of the $800 \times 600$ pixel object with a BER $=0.03$. As can be seen, there is a greater contrast between the white and black pixels and the edges are well-defined in the images.

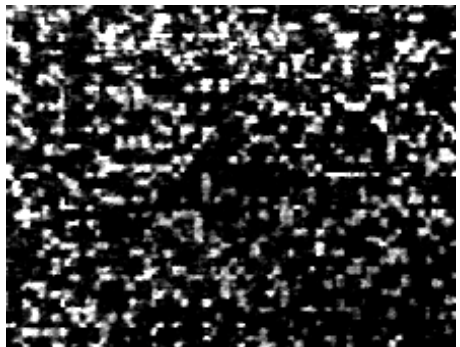

a)

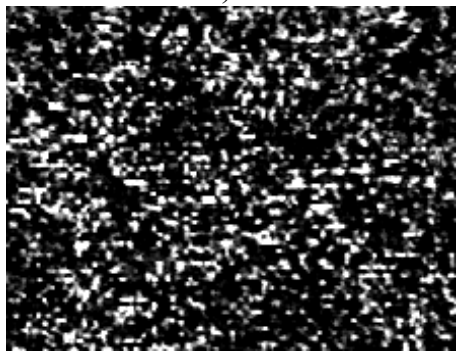

c)

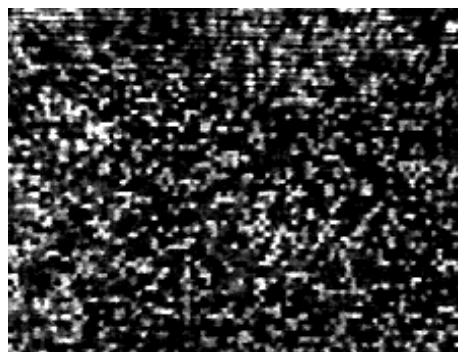

b)

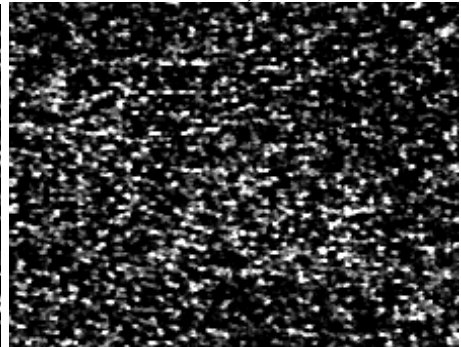

d)

Fig. 5: Best images obtained in an $80 \mu \mathrm{m}$ thick material for the objects a) $300 \times 300$ bits, b) 400x400 bits, c) 500x500 bits and d) $800 \times 600$ bits.

Therefore, we conclude that as the number of bits in the objects increases, the BER of the images also increases and the image quality decreases.

Moreover, we were able to store 25 holograms with a BER of less than 0.2 using HTM in an $80 \mu \mathrm{m}$ thick layer.

Considering the BER, holograms 1 to 8 have BER values below 0.1, and from hologram 10 on, BER values are around 0.1 . The $800 \times 600$ pixel object has values of BER close to 0.2 for holograms 15 to 25 .

\section{CONCLUSION}

In this study, four different data pages with a different pixel size were multiplexed in a PVA-acrylamide photopolymer. In order to reduce the zero frequency of the FT of the data pages, HTM was used to modulate the object beam which was introduced in the holographic set-up with a TN-LCD. Amplitude modulation produces a high zero frequency which may saturate the dynamic range of the material and limit the accessible dynamic range. If this zero frequency is reduced many holograms may be superimposed at the same position in the material. Once the holograms were stored by peristrophic multiplexing, the BER of the images was calculated to quantify the quality of the images as a function of the number of multiplexed holograms. With a thickness of $80 \mu \mathrm{m}$, about 22 holograms were stored with a high quality. This study shows the possibilities of this photopolymer as a holographic memory.

\section{ACKNOWLEDGEMENT}

This work was supported by the "Ministerio de Ciencia e Innovación" (Spain) under projects FIS2008-05856-C02-01 and FIS2008-05856-C02-02 and by the "Generalitat Valenciana" under projects GVPRE/2008/274 and GVPRE/2008/135. 


\section{REFERENCES}

[1] Gallego, S., Ortuño, M., García, C., Neipp, C., Beléndez, A., Pascual, I., "High-efficiency volume holograms recording on acrylamide and N,N ' methylene-bis-acrylamide photopolymer with pulsed laser," J. Mod. Opt. 52, 1575-1584, (2005).

[2] Gallego, S., Ortuno, M., Neipp, C., Marquez, A., Belendez, A., Pascual, I., "Characterization of polyvinyl alcohol/acrylamide holographic memories with a first-harmonic diffusion model," Appl. Opt. 44, 6205-6210, (2005).

[3] Hsu, K. Y., Lin, S. H., Hsiao, Y. N., Whang, W. T., "Experimental characterization of phenanthrenequinonedoped poly(methyl methacrylate) photopolymer for volume holographic storage," Opt. Eng. 42, 1390-1396, (2003).

[4] Schnoes, M., Ihas, B., Hill, A., Dhar, L., Michaels, D., Setthachayanon, S., Schomberger, G., Wilson, W. L., "Holographic data storage media for practical systems," in Practical Holography XVII and Holographic Materials IX, Tung H.Jeong, ed., Proc SPIE 5005, 29-37, (2003).

[5] Mok, F. H., Burr, G. W., Psaltis, D., "System metric for holographic memory systems," Opt. Lett. 21, 896-898, (1996).

[6] Ortuño, M., Gallego, S., García, C., Neipp, C., Beléndez, A., Pascual, I., "Optimization of a 1 mm thick PVA/acrylamide recording material to obtain holographic memories: method of preparation and holographic properties," Appl. Phys. B 76, 851-857, (2003).

[7] Lu, K. and Saleh, B. E. A., "Theory and design of the liquid-crystal TV as an optical spatial phase modulator," Opt. Eng. 29, 240-246, (1990).

[8] Yamauchi, M. and Eiju, T., "Optimization of twisted-nematic liquid-crystal panels for spatial light phase modulation," Opt. Comm 115, 19-25, (1995).

[9] Kim, H. and Lee, Y. H., "Unique measurement of the parameters of a twisted-nematic liquid-crystal display," Appl. Opt. 44, 1642-1649, (2005).

[10] Márquez, A., Iemmi, C., Moreno, I., Davis, J. A., Campos, J., Yzuel, M. J., "Quantitative prediction of the modulation behavior of twisted nematic liquid crystal displays based on a simple physical model," Opt. Eng. 40, 2558-2564, (2001).

[11] Guerrero, R. A., "Volume holographic storage and animation based on addressing with an elastomer phase mask," Opt. Comm. 245, 75-83, (2005).

[12] Joseph, J. and Waldman, D. A., "Homogenized Fourier transform holographic data storage using phase spatial light modulators and methods for recovery of data from the phase image," Appl. Opt. 45, 6374-6380, (2006).

[13] Remenyi, J., Varhegyi, P., Domjan, L., Koppa, P., Lorincz, E., "Amplitude, phase, and hybrid ternary modulation modes of a twisted-nematic liquid-crystal display at similar to $400 \mathrm{~nm}$," Appl. Opt. 42, 3428-3434, (2003).

[14] Renu, J., Joby, J., Kehar, S., "Holographc digital data storage using phase-modulated pixels," Opt. Laser Eng. 43, 183-194, (2005).

[15] Márquez, A., Gallego, S., Mendez, D., Alvarez, M. L., Fernández, E., Ortuño, M., Neipp, C., Beléndez, A., Pascual, I., "Accurate control of a liquid-crystal display to produce a homogenized Fourier transform for holographic memories," Opt. Lett. 32, 2511-2513, (2007).

[16] Jang, J. S. and Shin, D. H., "Optical representation of binary data based on both intensity and phase modulation with a twisted-nematic liquid-crystal display for holographic digital data storage," Opt. Lett. 26, 1797-1799, (2001).

[17] Domjan, L., Koppa, P., Szarvas, G., Remenyi, J., "Ternary phase-amplitude modulation with twisted nematic liquid crystal displays for Fourier-plane light homogenization in holographic data storage," Optik 113, 382-390, (2002).

[18] Márquez, A., Gallego, S., Méndez, D., Álvarez, M. L., Fernández, E., Ortuño, M., Beléndez, A., Pascual, I., "Characterization and optimization of liquid crystal displays for data storage applications," in Liquid crystals and applications in optics, M. Glogarova, P. PalffyMuhoray, and M. Copic, eds.,6587, 58715-58715, (2007).

[19] Dhar, L., Curtis, K., Tackitt, M., Schilling, M., Campbell, S., Wilson, W., Hill, A., Boyd, C., Harris, A., "Holographic storage of multiple high-capacity digital data pages in thick photopolymer systems," Opt. Lett. 23, 1710-1712, (1998). 
[20] Varhegyi, P., Koppa, P., Ujhelyi, F., Lorincz, E., "System modeling and optimization of Fourier holographic memory," Appl. Opt. 44, 3024-3031, (2005).

[21] Coufal, H., Psaltis, D., Sincerbox, G. T., Holographic Data Storage, Springer-Verlag, New Cork, (2000).

[22] Fernández, E., Ortuño, M., Gallego, S., García, C., Beléndez, A., Pascual, I., "Comparison of peristrophic multiplexing and a combination of angular and peristrophic holographic multiplexing in a thick PVA/acrylamide photopolymer for data storage," Appl. Opt. 46, 5368-5373, (2007).

[23] Fernández, E., Ortuño, M., Gallego, S., García, C., Márquez, A., Beléndez, A., Pascual, I., "Multiplexing holograms for data page storage as a holographic memory in a PVA/AA photopolymer," in Practical Holography Xxii: Materials and Applications, H. Bjelkhagen and R. Kostuk, eds., Proc. SPIE 6912, 91214, (2008).

[24] Fernández, E., Ortuño, M., Gallego, S., Márquez, A., García, C., Fuentes, R., Beléndez, A., Pascual, I., "Optimization of a holographic memory setup using an LCD and a PVAbased photopolymer," Optik, (2009).

[25] Márquez, A., Campos, J., Yzuel, M. J., Moreno, I., Davis, J. A., Iemmi, C., "Characterization of edge effects in twisted nematic liquid crystal displays," Opt. Eng. 39, 3301-3307, (2000).

[26] Fernández, E., Ortuño, M., Gallego, S., Márquez, A., García, C., Beléndez, A., Pascual, I., "Multiplexed holographic data page storage on a PVA/acrylamide photopolymer memory," Appl. Opt. 47, 4448-4456, (2008). 